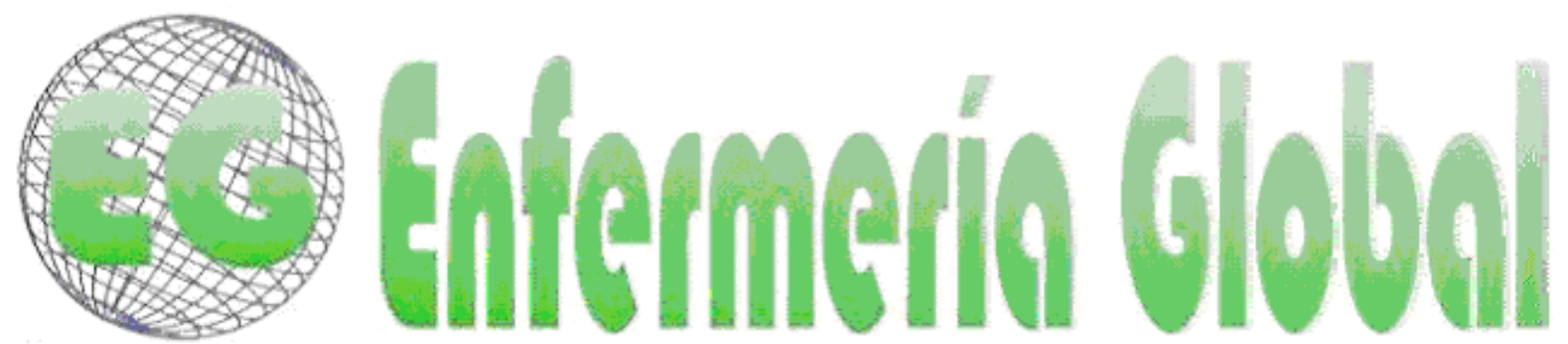

\title{
Revisión de evidencias sobre la influencia de la dieta en la evolución de la EPOC
}

Review of evidence on the influence of the diet on the evolution of COPD.

\author{
*De los Santos Izquierdo, JM., *Peña Molinero, C., *Ortega Barriuso, R., \\ *Manso Melgosa, AB.
}

\section{*Enfermero/a. Complejo Asistencial Universitario de Burgos. E-mail: jsantosi@saludcastillayleon.es}

Palabras clave: EPOC; dieta.

Keywords: COPD; diet.

\section{RESUMEN}

La Enfermedad Pulmonar Obstructiva Crónica (EPOC) ocasiona una importante morbimortalidad, con un gran consumo de recursos sanitarios. La causa principal es el consumo de tabaco. La EPOC es evitable y aunque no es curable, sí es susceptible de mejoría en términos de calidad de vida y aumento de la supervivencia, utilizando los recursos terapéuticos disponibles entre los que se encuentra la rehabilitación y la educación sanitaria.

La rehabilitación incluye programas de entrenamiento cuyo fin es mejorar la disnea, el cansancio y la tolerancia al ejercicio. Se compone de programas de entrenamiento físico, educación sanitaria, intervención psicológica, intervención sobre el comportamiento y educación sobre dieta y nutrición.

En el presente trabajo se realizó una recopilación bibliográfica de los artículos publicados en los últimos cinco años sobre la educación dietética a los pacientes con EPOC. Se usó la herramienta de lectura crítica CASPE, para seleccionar los de mayor calidad metodológica, con el objetivo de revisar los estudios existentes sobre la influencia de la dieta en los pacientes diagnosticados de EPOC, evaluar las evidencias existentes e incorporarlos a la pauta de actuación ante este tipo de pacientes.

La conclusión de esta revisión es que los pacientes con EPOC podrían beneficiarse de una dieta rica en hortalizas, frutas y verduras, soja, fibra y aceite de oliva; mientras que las dietas ricas en azúcares y carnes curadas podrían empeorar su enfermedad. Aunque se recomienda realizar estudios complementarios que corroboren dichas afirmaciones.

\section{ABSTRACT}

Chronic Obstructive Pulmonary Disease (COPD) causes significant morbidity and mortality, with a large consumption of health resources. The main cause is smoking. Despite the fact there is no cure for COPD, it can be avoided resulting in an improvement in the quality of life and an increase the survival rate, through available therapeutic resources, among which are the rehabilitation and health education. 
Rehabilitation includes training programs designed to improve dyspnoea, fatigue and exercise tolerance. It consists of physical training programs, health education, psychological intervention, conduct intervention and education on diet and nutrition.

This study has been performed on an extensive bibliographic compilation of published articles on dietary education patients with COPD in the last five years. In order to get the highest methodological quality articles and perform a review of the existing studies on the influence of diet in patients diagnosed with COPD and evaluate the evidence of those patients, it was necessary to implement a critical reading tool (CASPE), to incorporate them into the pattern of performance against these patients.

The conclusion of this review is that patients with COPD may profit from a diet rich in fruit, vegetables, soy, fiber and olive oil, while a diet rich of sugars and cured meat may worsen your condition. However additional studies to corroborate these claims are recommended.

\section{INTRODUCCIÓN}

La enfermedad pulmonar obstructiva crónica (EPOC) se caracteriza por una limitación crónica al flujo aéreo, no totalmente reversible y lentamente progresiva. Se asocia con una respuesta inflamatoria pulmonar anómala a las partículas de gases nocivos ${ }^{1}$. La EPOC es asintomática en sus fases iniciales. Cuando progresa, suele cursar con disnea, tos y expectoración. La EPOC es una enfermedad prevenible y tratable. En ella se incluyen: la bronquitis crónica que se define por la presencia de tos y expectoración diaria durante al menos 3 meses al año en 2 años consecutivos, tras haberse descartado otras causas de tos productiva y el enfisema (dilatación permanente de los espacios aéreos distales a los bronquiolos terminales), con destrucción de la pared alveolar y sin fibrosis manifiesta.

Los programas educativos para promover el auto-cuidado se han dirigido a: la deshabituación tabáquica, mejorar el ejercicio, nutrición, auto-tratamiento de las agudizaciones, técnicas de inhalación o adaptación a las actividades de la vida diaria. Cuando se ha analizado el papel del auto-cuidado en la evolución de la EPOC, no se han encontrado evidencias concluyentes sobre el impacto de dichas actividades ${ }^{2}$. Es posible que la adquisición de habilidades y conocimientos no implique directamente un cambio de conducta o, quizás, dado que las intervenciones en la EPOC son multifactoriales sea difícil individualizar los efectos de uno de los componentes. Así, en el impacto de la hospitalización a domicilio, la educación del paciente juega un papel muy importante junto con otros aspectos de la intervención, como la visita domiciliaria o la accesibilidad al equipo ${ }^{3}$. Hay trabajos que sugieren que la educación para promover el auto-cuidado reduce la medicación de rescate durante las agudizaciones de la EPOC ${ }^{4}$ o el uso de recursos sanitarios ${ }^{5}$.

Se estima que los pacientes con EPOC presentan una media de dos agudizaciones al año, aunque éstas son menos frecuentes en la EPOC leve y mucho más habituales en los casos más graves. En España se estima que un médico de Atención Primaria, atiende al año a unos 75 pacientes con agudización de la EPOC/bronquitis crónica, lo que supone el $3,5 \%$ de todas las infecciones atendidas y el $7 \%$ de las infecciones respiratorias ${ }^{6}$.

\section{Etiología de la EPOC}

Fumar es el factor de riesgo más importante para desarrollar EPOC. Ser fumador pasivo es otro factor de riesgo junto con los relacionados con la exposición 
medioambiental. Otro grupo de factores de riesgo son los aspectos genéticos y la hiperreactividad bronquial.

La causa más frecuente de agudización de la EPOC es la infección del tracto respiratorio inferior (50-70\%, la mayoría de origen bacteriano).

\section{Epidemiología de la EPOC}

La EPOC es más frecuente en hombres, en la edad avanzada, en fumadores y en personas de bajo nivel educativo.

La prevalencia en España según el estudio IBERPOC ${ }^{7}$, realizado en 1996-1997 entre individuos de 40 a 69 años, fue del 9,1\% (14,3\% en varones y 3,9\% en mujeres; $15 \%$ en fumadores, $12,8 \%$ en ex fumadores y $4,1 \%$ en no fumadores).

El estudio EPISCAN (Epidemiologic Study of COPD in Spain), realizado en 2006$2007^{8}$, ha cuantificado la prevalencia de EPOC en la población española entre 40 y 80 años en el $10,2 \%(15,1 \%$ en hombres y $5,7 \%$ en mujeres; $39,9 \%$ en hombres fumadores, $18 \%$ en ex fumadores y $7,6 \%$ en no fumadores vs. $15,4 \%, 6 \%$, y $5,5 \%$, respectivamente, en el caso de las mujeres).

Esta patología ocasiona una importante morbi-mortalidad entre sus portadores, con un gran consumo de recursos sanitarios y es la causa del $38 \%$ de las incapacidades laborales secundarias a la enfermedad ${ }^{9}$.

A nivel mundial, la prevalencia poblacional de EPOC se estima en torno al 1\%. Según la Encuesta Nacional de Salud de 2006, en España fuma el 29,5\% de la población $(35,33 \% \text { de los varones y } 23,88 \% \text { de las mujeres })^{10}$.

A partir de una cierta edad, la función respiratoria va disminuyendo y se observa una caída del FEV1 (volumen de aire que se expulsa durante el primer segundo de la

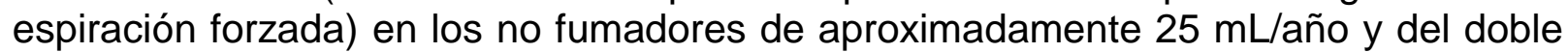
entre los fumadores susceptibles.

Según el estudio Global Burden of Disease (Carga global de morbilidad) realizado bajo el auspicio de la Organización Mundial de la Salud y del Banco Mundial, la EPOC es actualmente la cuarta causa principal de muerte en el mundo, con 2,75 millones de muertes y se prevé un aumento adicional de la mortalidad en los próximos años ${ }^{11}$.

\section{Diagnóstico de la EPOC}

Se debe sospechar EPOC en todo individuo mayor de 40 años que haya fumado (más 10 paquetes/año) y que presente síntomas compatibles con la enfermedad (tos, expectoración, disnea de esfuerzo). Entre ese grupo de población la prevalencia de EPOC es superior al $30 \%{ }^{1}$.Para confirmar el diagnostico se debe realizar una espirometría, post-broncodilatación y fuera de la fase de agudización.

La espirometría, además de permitir el diagnóstico de la EPOC y ayudar a valorar su evolución, se ha mostrado útil para mejorar el resultado del tratamiento de la deshabituación del tabaquismo ${ }^{12}$. 
La atención a los pacientes con EPOC debe ser abordada desde diferentes niveles de asistencia sanitaria, con una implicación multidisciplinar de los profesionales sanitarios.

La EPOC es una enfermedad evitable, y aunque no es curable, sí es susceptible de mejoría en términos de calidad de vida y aumento de la supervivencia utilizando en la forma adecuada los recursos terapéuticos disponibles.

\section{Algoritmo diagnóstico de la EPOC}

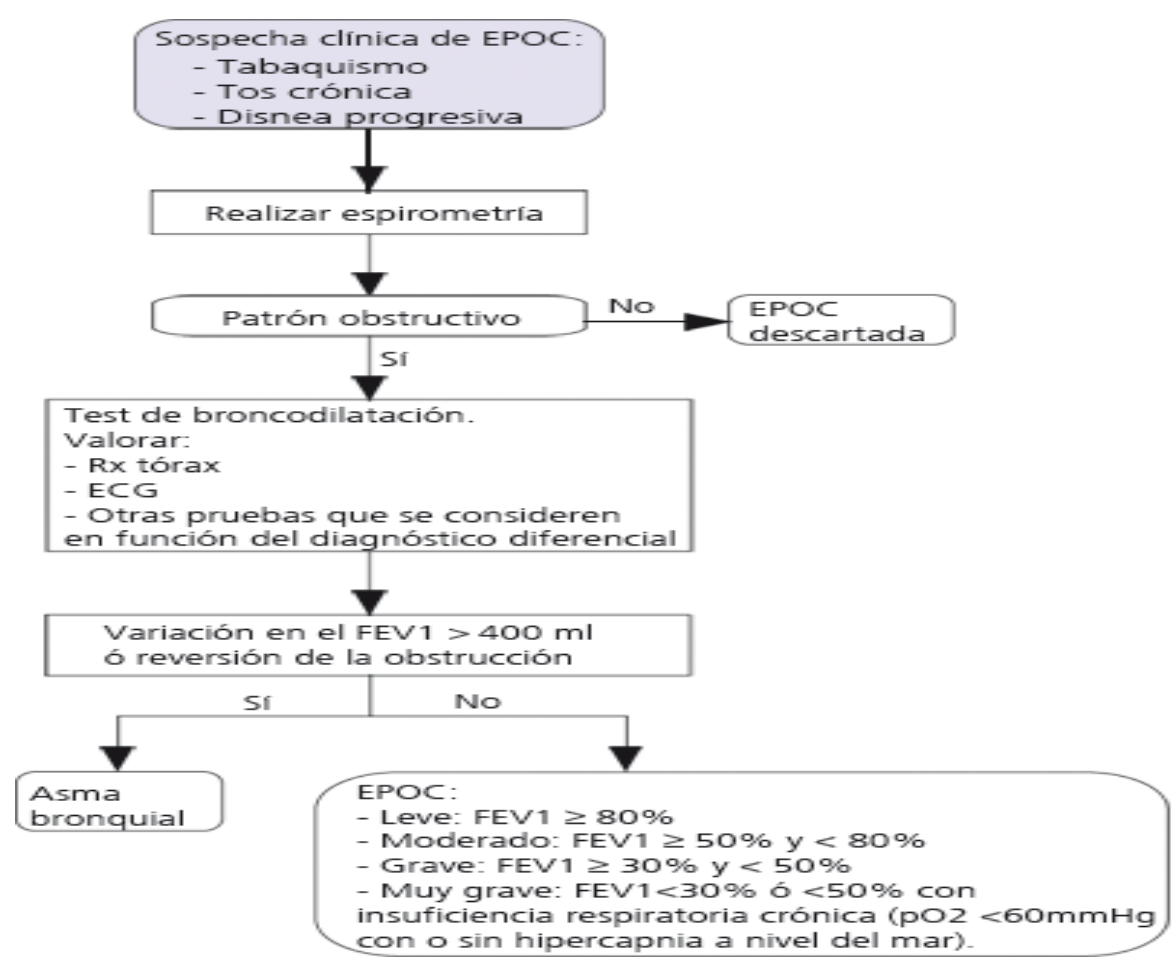

\section{Tratamiento de la EPOC}

- Reducción de los factores de riegos.

- Tratamiento farmacológico con el fin de prevenir y controlar los síntomas, reducir la frecuencia y severidad de las exacerbaciones. La vacunación antigripal reduce aproximadamente el $50 \%$ las formas graves y muerte por EPOC (nivel de recomendación A) ${ }^{9}$.

- Tratamiento no farmacológico:

- Rehabilitación. Ya que los pacientes con EPOC parecen beneficiarse de los programas de entrenamiento mejorando la tolerancia al ejercicio y los síntomas de disnea y cansancio. Dentro de la rehabilitación se encuentran programas de entrenamiento físico, educación, nutrición, intervención psicológica e intervención sobre el comportamiento ${ }^{9}$. 
- La hospitalización a domicilio y otros programas de atención no hospitalaria pueden ser utilizados como alternativa para el tratamiento de las exacerbaciones (nivel de recomendación A) ${ }^{9}$.

Dado que la EPOC es una enfermedad crónica que aunque es prevenible no tiene curación, la actuación ante estos pacientes debe centrarse en medidas terapéuticas, rehabilitación pulmonar y educación sanitaria encaminadas a mejorar la disnea y la calidad de vida de este tipo de pacientes

El objetivo de esta revisión bibliográfica fue revisar los estudios existentes sobre la influencia de la dieta en los pacientes diagnosticados de EPOC, evaluar las evidencias e incorporarlos a la pauta de actuación ante este tipo de pacientes.

\section{MATERIAL Y MÉTODO}

Para la realización de la presente revisión bibliográfica se ha partido de una hipótesis que posteriormente dio pie a confeccionar la pregunta clínicamente contestable con formato PICO de la cual se extrajo los Mesh (Medical Subject Headings), tesauro (lenguaje controlado) usado para indesar los articulos en Pubmed y Decs (Descriptores en Ciencias de la Salud), tesauro usado para indesar literatura científica en las fuentes de información disponibles en la Biblioteca Virtual en Salud.

Una pregunta es un interrogante, es por lo tanto la primera etapa de cualquier proceso de investigación. La pregunta con estructura PICO, es una manera estructurada de formular preguntas clínicas, susceptibles de respuesta.

Lo que hacemos con este proceso es descomponer nuestra inquietud en diferentes unidades, de tal manera que la suma de las mismas definan de forma explícita nuestro problema y nos sirvan de orientación para la búsqueda de respuestas.

Las unidades fundamentales de una pregunta con esta estructura son:

- Paciente o problema clínico (P). Determina el objeto de la pregunta.

- Intervención (I). Es la acción, que vamos a analizar.

- Comparación (C). Es la equiparación entre diferentes intervenciones, para valorar si mejora nuestros resultados. No siempre es necesario incluirla.

- Resultado, outcome (O). Es el fin que se persigue. De tal manera que estos deben ser relevantes clínica, social o económicamente y siempre deben ser medibles.

La Hipótesis de trabajo fue la siguiente: ¿Cómo influye la dieta en la evolución de la EPOC?, ¿Obtendría el paciente diagnosticado de EPOC un beneficio para su vida diaria de una educación sanitaria que incluyese consejos dietéticos?

El siguiente paso consistió en convertir esta inquietud en una pregunta tipo PICO: 


\begin{tabular}{|l|l|l|l|}
\hline \multicolumn{1}{|c|}{ Paciente (P) } & Intervención (I) & Comparación (C) & \multicolumn{1}{|c|}{ Outcome (O) } \\
\hline $\begin{array}{l}\text { Paciente } \\
\text { diagnosticado de } \\
\text { EPOC }\end{array}$ & $\begin{array}{l}\text { Consejos } \\
\text { dietéticos e } \\
\text { influencia de la } \\
\text { dieta }\end{array}$ & $\begin{array}{l}\text { Frente a no incluir } \\
\text { en la educación } \\
\text { sanitaria los } \\
\text { consejos } \\
\text { dietéticos }\end{array}$ & $\begin{array}{l}\text { Mejorar la calidad } \\
\text { de vida del } \\
\text { paciente con } \\
\text { EPOC }\end{array}$ \\
\hline
\end{tabular}

La pregunta podría reflejarse de la siguiente manera: ¿Los pacientes con EPOC podrían beneficiarse de una educación sanitaria que incluyesen consejos dietéticos para mejorar su calidad de vida? De esta pregunta se obtuvieron las palabras clave, los Mesh y Decs correspondientes como queda reflejado en la siguiente tabla:

\begin{tabular}{lll}
\multicolumn{1}{c}{$\begin{array}{c}\text { Lenguaje } \\
\text { natural }\end{array}$} & \multicolumn{1}{c}{ Mesh } & \multicolumn{1}{c}{ Decs } \\
\hline EPOC & COPD & $\begin{array}{l}\text { Pulmonary Disease, Chronic } \\
\text { Obstructive }\end{array}$ \\
\hline Dieta & $\begin{array}{l}\text { Diet. } \\
\text { Subheadings:-Education } \\
\text { - Nursing }\end{array}$ & Diet \\
& \multicolumn{2}{c}{} \\
\hline
\end{tabular}

Con estos Mesh y Decs se realizó la búsqueda bibliográfica en las siguientes bases de datos: Pubmed, Medline on Ovidop, Web of Knowledge (Wok), Cochrane plus, Trip data base, JBI Connect, Cinahl y Cuiden. Posteriormente se realizó una revisión de los artículos publicados desde el año 2006 no usando ninguna otra limitación.

Se evaluó la calidad metodológica de cada estudio seleccionado mediante la herramienta de lectura crítica CASPE $^{13}$.

\section{RESULTADOS Y DISCUSIÓN}

En la revisión localizamos 1006 artículos seleccionando inicialmente 47 por su calidad metodológica, aunque utilizamos los que más nivel de evidencia tenían, un total de 12, según el esquema siguiente: 


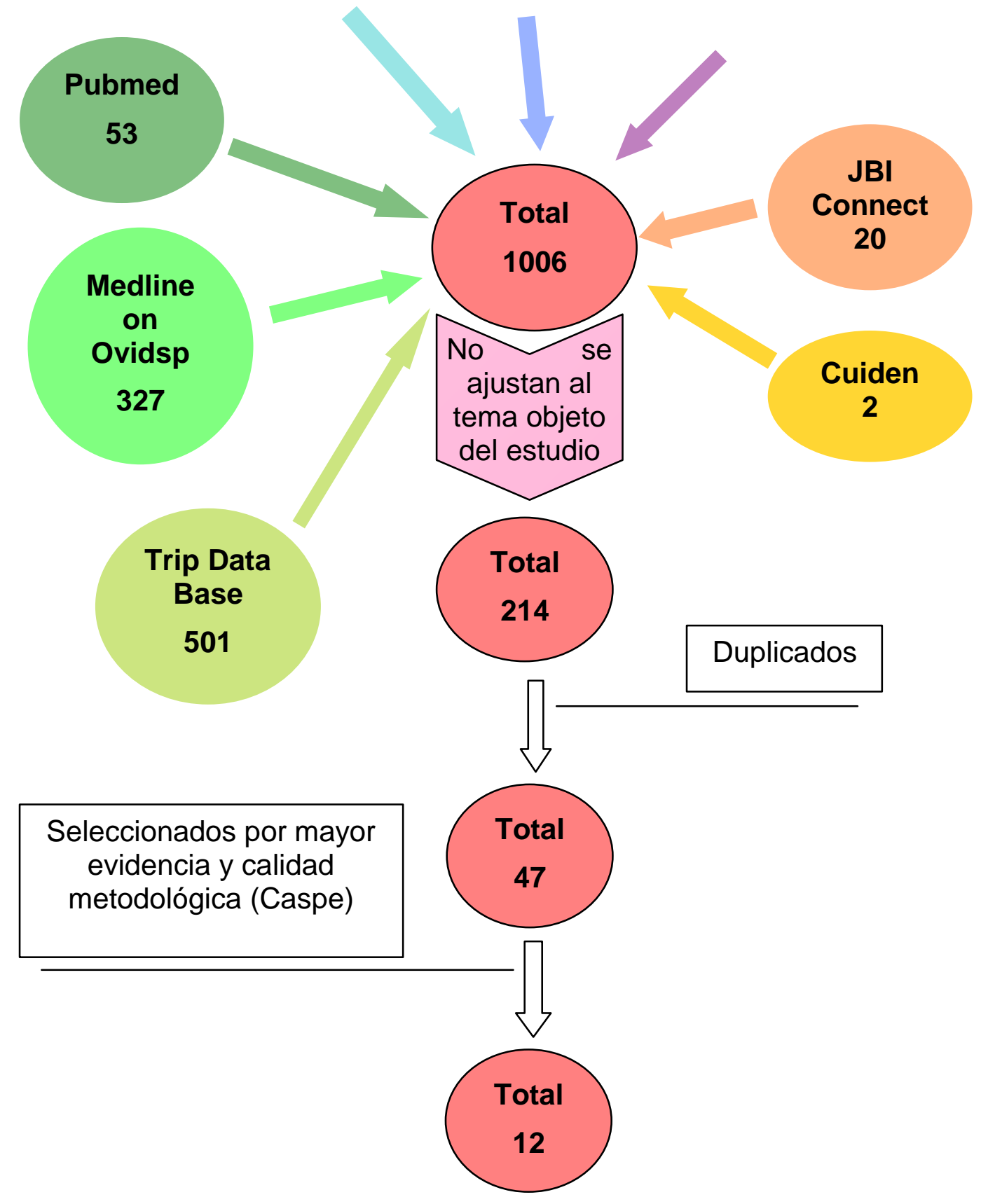

E. Keranis y colaboradores ${ }^{14}$ realizaron un estudio con el objetivo de demostrar la asociación entre una dieta rica en alimentos antioxidantes y la función pulmonar en pacientes con EPOC. Durante tres años hizo un seguimiento a 120 pacientes diagnosticados de EPOC divididos en dos grupos uno cuya dieta era rica en frutas y vegetales y el otro con una dieta libre.

La actividad física, la ingesta de alcohol, las enfermedades asociadas o el hecho de que el $19 \%$ de los pacientes dejara de fumar durante el estudio fueron desestimadas como factores de confusión ya que no existieron diferencias significativas entre los dos grupos.

En cuanto a las limitaciones del presente estudio se destacan que el estrés oxidativo no se evaluó usando muestras de sangre, orina o aire exhalado. La otra limitación fue que las exacerbaciones se midieron por los síntomas reflejados en el cuestionario 
rellenado por los propios pacientes, lo que puede ser la causa de que el número de exacerbaciones fuese menor que los reflejados en estudios similares a este.

Este estudio constató que una dieta rica en frutas y verduras puede tener un impacto significativo en la función pulmonar y que futuros estudios podrían demostrar la asociación o no entre una dieta rica en antioxidantes y la mejoría de la función pulmonar.

En el estudio realizado en la población japonesa por Fumi Hirayama y colaboradores ${ }^{15}$ estos relatan la relación de la dieta y el riesgo de padecer EPOC. El primero tiene como objetivo valorar la relación entre el consumo de productos ricos en soja, el riesgo de padecer EPOC y la prevalencia de síntomas respiratorios. Para ello se llevó a cabo un estudio con un total de 278 pacientes en el grupo de casos y 340 en el grupo de control. A todos los pacientes se les realizaron estudios para determinar su función respiratoria, estudios antropomórficos, características de su estilo de vida y composición de su dieta habitual mediante la realización de un cuestionario estructurado y validado para tal fin.

En cuanto a las limitaciones, cabe destacar, que la evaluación de la dieta se realizó mediante el cuestionario que el propio autor pasó a los interesados y familiares para, en la medida de lo posible, mejorar la tasa y exactitud de las respuestas, incluso en individuos con problemas de memoria. La otra limitación expuesta por los autores fue que los participantes del grupo de control fueron elegidos por propia voluntad, viéndose en la obligación de dejar de consumir productos ricos en soja, siendo los propios autores los que excluyeron ciertos productos, como la sopa de miso y la salsa de soja, por no poder cuantificar con exactitud la concentración de soja de los mismos.

La evidencia de los efectos beneficiosos de los productos de soja debe ser corroborada con otros estudios en los que el número de mujeres participantes sea mayor y en poblaciones diferentes a la japonesa.

C.E. Weekes y colaboradores ${ }^{16}$ realizaron su estudio con el objetivo de demostrar el efecto del asesoramiento dietético y el aporte de suplementos dietéticos en pacientes con EPOC. En el estudio participaron 36 personas en el grupo de la intervención y 30 en el de control, de los cuales 59 completaron los primeros seis meses y 37 terminaron el estudio. Se midió el efecto de la intervención no solo en la mejora de los problemas nutricionales sino también sobre las medidas objetivas y subjetivas del estado funcional. Además midió los efectos una vez que cesó la intervención y a los seis meses de haber comenzado el estudio.

El principal sesgo fue el cegamiento inadecuado tanto para la asignación como para la evaluación de los resultados. Para reducirlo al mínimo los autores analizaron los resultados al final del estudio, y la dieta fue confeccionada por dos especialistas que estuvieron cegados tanto al grupo de tratamiento como al de los resultados.

El asesoramiento dietético dio lugar a importantes beneficios en la ingesta de la dieta, la composición corporal, la calidad de vida y las medidas subjetivas del estado funcional. Los pacientes del grupo de intervención ganaron aproximadamente $2 \mathrm{~kg}$ de peso durante el periodo de la intervención y el peso se mantuvo durante el periodo de seguimiento. Las posibles razones de esta mejora fueron el hecho de que el 
asesoramiento dietético fue individualizado según las necesidades de cada paciente. Estos resultados están en la misma línea que estudios similares a este.

El estudio de Rui Jiang y colaboradores ${ }^{17}$ pretendía demostrar la relación entre el consumo de carne curada y el riesgo de padecer EPOC en mujeres, para lo cual se realizó un estudio a una población de 121700 mujeres con edades comprendidas entre 38 y 63 años durante un periodo de seguimiento de 16 años.

En cuanto a los posibles sesgos, se destacó el método seguido para diagnosticar la EPOC y la exposición al humo; este último factor fue desechado ya que se tuvo en cuenta la condición de fumador, el número de años de fumador, el número de paquetes/día consumidos y la exposición al humo ambiental en el hogar y el trabajo.

La asociación entre ingesta de carne curada y EPOC puede estar relacionado con los efectos nocivos de los nitritos que contienen estas carnes, siendo estas el principal origen de los mismos.

Como puntos fuertes se destacan el gran tamaño de la cohorte y que tuvieron en cuenta factores dietéticos beneficiosos como la ingesta de pescado, frutas y verduras.

Se demostró que la frecuencia en el consumo de carne curada se asoció positivamente con el riesgo de ser diagnosticada de EPOC. Esta asociación es más fuerte en los exfumadores que en los que son fumadores activos. No se observó asociación entre los que nunca han fumado, probablemente debido al pequeño número de pacientes no fumadores que padecen EPOC.

En los estudios de Raphaëlle Varraso y colaboradores ${ }^{18}$ se intentó evaluar la relación entre la el consumo de carne curada y el riesgo de padecer EPOC en la población masculina; para ello efectuaron un seguimiento a 42915 hombres americanos durante 12 años, obteniendo una asociación entre el consumo de embutidos y el riesgo de padecer EPOC. Este hallazgo se achaca al contenido en nitritos que tienen este tipo de alimentos.

El estudio tiene varias limitaciones como son que la asociación entre la ingesta de embutidos y el diagnóstico de EPOC pueda deberse a un factor de confusión por el consumo de cigarrillos o la exposición al humo del tabaco. Para minimizar este posible sesgo los autores realizaron múltiples medidas de valoración de esta exposición.

Otro posible sesgo de este estudio fue el método diagnóstico de nuevos casos de EPOC. Finalmente, los autores del presente estudio detectaron la falta de una medida específica para cuantificar los niveles de ingesta de nitritos en la dieta.

En el segundo ${ }^{19}$, realizado durante un periodo de 16 años a una población de 72043 mujeres estadounidenses, se encontró una asociación entre las mujeres que tienen un patrón alimenticio rico en carne curada y roja, patatas fritas, postres y dulces y el riesgo de padecer EPOC.

En cuanto a los factores de confusión que detectaron los autores fueron el hábito de fumar, la exposición al humo del tabaco y el proceso seguido para el diagnostico de nuevos casos de EPOC. 
Raphaëlle Varraso y colaboradores ${ }^{20}$ intentaron probar la relación entre los hábitos alimenticios y la EPOC en hombres y mujeres americanos. Para ello, realizaron un estudio durante 12 años a una población de 51529 americanos con edades comprendidas entre los 40 y los 75 años. Analizaron dos tipos de dietas una tipo I con alto consumo en frutas, verduras y pescado y otra tipo II con alto contenido en carnes curadas y rojas, postres, dulces y patatas fritas. Se encontró que existe un riesgo importante de padecer EPOC entre los que consumían una dieta del tipo II.

Los estudios sobre la asociación de distintos alimentos y nutrientes y la EPOC han sugerido un efecto beneficioso de los antioxidantes, especialmente de la vitamina $C$ no detectándose una asociación clara entre un alimento particular y la EPOC.

Estudios sobre el efecto de los $\beta$-carotenos, el retinol y los suplementos de vitamina A, no mostraron cambios en los síntomas respiratorios y la función pulmonar; la causa podría ser que el efecto de cualquiera de ellos aisladamente es demasiado pequeño para poderse detectar.

El estudio realizado en el ámbito estadounidense por Raphaëlle Varraso ${ }^{21}$ sobre un grupo de 40215 personas con edades comprendidas entre 40 y 75 años y un seguimiento durante 12 años demostró que el riesgo de padecer enfermedades respiratorias y especialmente la EPOC disminuía entre las personas que tienen una dieta rica en fibra y más específicamente entre los que consumían la fibra de los cereales.

Las limitaciones descritas por los propios autores son: la metodología usada para diagnosticar la EPOC, los factores de confusión residuales como el tabaquismo, y por último, la dificultad de estudiar los efectos sobre la salud de un determinado nutriente por las complejas interrelaciones entre la ingesta de los diferentes componentes de la dieta.

Rosenkranz y colaboradores ${ }^{22}$ intentaron demostrar si la dieta con alto contenido en grasa aumentaría la inflamación de las vías aéreas y produciría una disminución en la función pulmonar en sujetos sanos.

Seleccionaron 20 personas adultas, 10 hombres y 10 mujeres, sin historia de enfermedad crónica y con una dieta baja en ingesta de frutas y verduras (menos de tres piezas por día). Se excluyeron a los fumadores, a los que estaban siguiendo alguna dieta de adelgazamiento y a los que estaban en tratamiento médico. También se excluyeron a los que tenían glucemias con valores $\geq 100$ pasadas 12 horas de la ingesta y tensiones arteriales $\geq 140 / 95$.

Los seleccionados se abstuvieron de tomar alcohol y de hacer ejercicio 24 horas antes, de tomar cafeína 12 horas y estuvieron en ayuno o tomaron una comida muy baja en grasa antes del estudio.

Se les realizaron pruebas antes y dos horas después de haber comido una dieta rica en grasas para medir su función pulmonar y su composición corporal, y pruebas analíticas para determinar los valores del colesterol, los triglicéridos y la proteína Creactiva. 
En este estudio demostraron que una dieta rica en grasas produce un aumento significativo en los niveles de colesterol, triglicéridos y óxido nítrico exhalado, aunque no se vio ningún cambio en la proteína C-reactiva.

No se pudo demostrar que estos cambios fueran mayores en los sujetos con niveles más altos de grasa corporal y tampoco que se produjese una disminución de la función pulmonar.

Las limitaciones destacadas en este estudio son que dentro de las pruebas de la función pulmonar no se evaluó la hiperreactividad de la vía aérea y que el análisis se centró en la respuesta a una sola ingesta, constatando que existen estudios que reflejan que después de 12 semanas siguiendo dietas ricas en grasas es cuando se producen cambios en la vía aérea.

El aumento de los niveles de óxido nítrico exhalado y el aumento de la proteína Creactiva según otros estudios, se ve alterada pasadas 24 horas mientras que, en éste la medición fue a las dos horas. En cuanto al aumento de los triglicéridos podría deberse al alto contenido en hidratos de carbono de los helados de crema que han compuesto la dieta empleada para el presente estudio; por ello sería importante poder repetir estudios con dietas con alto contenido en grasas sin alto contenido en hidratos de carbono.

Los resultados sugieren que una dieta rica en grasas y tal vez en hidratos de carbono, puede contribuir a la inflamación crónica de las vías aéreas y por lo tanto al riesgo de desarrollar EPOC.

Hirayama Fumi y colaboradores ${ }^{23}$ realizaron su estudio con una muestra de 278 para el grupo de intervención y 340 para el grupo de control, con el objetivo de comprobar si la ingesta de ácido fólico en la dieta tiene un efecto beneficioso sobre la función pulmonar, en la población japonesa.

En cuanto a los sesgos, se destaca que los participantes en el estudio eran voluntarios aunque permanecieron cegados al tema del estudio, y que el método, consistió en la cumplimentación de un cuestionario en colaboración con el autor principal del estudio.

Jordi de Batlle y colaboradores ${ }^{24}$, realizaron estudio sobre 267 pacientes con EPOC cuya finalidad era evaluar la relación entre alimentos ricos en nutrientes antioxidantes y marcadores séricos de estrés oxidativo en la EPOC.

Este estudio presenta varias limitaciones, como son que la ingesta alimentaria está documentada únicamente a través de encuestas validadas para tal fin. La segunda fue la transversalidad del estudio, con lo cual no se tiene información sobre el ordenamiento temporal y la posible causalidad, aunque ellos consideran, que lo más probable fue que el estrés oxidativo fuera modificado por la dieta. Y por último que los hallazgos del estudio están limitados porque en el grupo había pacientes fumadores, lo que es compatible con una mayor demanda de antioxidantes. Los resultados de su estudio fueron: una asociación entre el consumo de vitamina $E$ y los niveles séricos de carbonilos en pacientes diagnosticados de EPOC y fumadores.

En segundo lugar, la asociación entre el consumo de aceite de oliva y el aumento de los niveles séricos de GSH en pacientes diagnosticados de EPOC y fumadores. Este 
resultado esta avalado por el conocimiento actual sobre los componentes del aceite de oliva, que incluso hace posible que este consumo pueda incrementar la producción endógena de antioxidantes. Además, hay estudios experimentales que sugieren que bajas concentraciones de flavonoides presentes en el aceite de oliva pueden estimular la transcripción de un gen esencial para la síntesis de GSH.

Otro resultado obtenido fue la asociación inversa entre el consumo de vegetales y los niveles de malondialdehído sérico en exfumadores.

A diferencia de lo que se esperaba no se encontraron asociaciones de otros alimentos ricos en antioxidantes. Como posible explicación, se sugiere el consumo relativamente alto de antioxidantes en los sujetos que formaban parte del presente estudio. Otra explicación aportada por los autores es la dificultad para relacionar el promedio de la ingesta diaria y los niveles exactos de estrés oxidativo, ante la variabilidad diaria en la ingesta de cualquier nutriente y la vida media de los marcadores de estrés oxidativo.

Por el contrario, no pudieron encontrar una explicación a la asociación entre la ingesta de vegetales y el malondialdehído solo en los ex fumadores.

Este estudio sugiere que, además de la importancia de reforzar las iniciativas antitabaco, las recomendaciones de aumentar en la dieta el consumo de antioxidantes pueden tener efectos beneficiosos en paciente con EPOC.

Carel $\mathrm{R}$ y colaboradores ${ }^{25}$ realizaron un estudio con el fin de comprobar si el aporte de suplementos dietéticos junto con un programa de rehabilitación respiratoria reducía las exacerbaciones y los ingresos de pacientes con EPOC. Para ello obtuvieron una muestra de 39 pacientes con EPOC, pérdida de masa muscular y moderada obstrucción al flujo aéreo de estos 23 fueron asignados al grupo de intervención y 16 al grupo control. Analizaron la masa muscular y la capacidad de ejercicio a los cuatro, 12 y 24 meses.

Las conclusiones a las que llegaron los autores fueron que a los cuatro meses mejoraban los parámetros estudiados pero la eficiencia de las medidas era baja. Mientras que a los 24 meses aunque no observaron diferencias en el número de exacerbaciones, sí en la gravedad de las mismas lo que redujo de forma importante los costes. En este estudio no pudieron dilucidar si estos beneficios eran producidos por el aporte de suplementos nutricionales o por la rehabilitación respiratoria aisladamente.

\section{CONCLUSIONES}

Esta revisión bibliográfica tiene como fin comprobar el conocimiento existente sobre la influencia de la dieta en pacientes con EPOC. Partiendo de la base de que la EPOC es incurable, todo el esfuerzo terapéutico debe ir encaminado hacia mejorar la calidad de vida y disminuir las exacerbaciones, con la consiguiente reducción del gasto sanitario.

De los estudios revisados se desprende que las dietas ricas en alimentos antioxidantes, frutas y verduras, fibra, especialmente la fibra de los cereales, y el consumo de aceite de oliva mejoran la función pulmonar y por lo tanto la calidad de vida de los pacientes con EPOC. 
Dos estudios señalan el beneficio sobre la función pulmonar de la ingesta de soja y de alimentos ricos en ácido fólico. Por el contrario, otros estudios han demostrado que la ingesta de carnes curadas, patatas fritas, dulces y dietas con alto contenido en grasas tiene un efecto perjudicial sobre la función pulmonar.

Otro trabajo que relaciona la educación nutricional, el aporte de suplementos nutricionales en la dieta y la realización de ejercicio en pacientes con EPOC, demuestra la eficiencia de estas medidas a partir de los 24 meses de terapia.

Todas estas evidencias deben ser corroboradas con más estudios que subsanen los sesgos detectados, para de esta manera tener la certeza de su utilidad en la práctica diaria.

\section{BIBLIOGRAFÍA}

1. Martín-Escudero JC. Conducta a seguir ante la EPOC desde Medicina Interna.Rev Clin Esp 2010; 210(4):171-177.

2. Perpiñá $M$, Sobradillo $V$, Castillo J, Duce $F$, et al. Búsqueda de información y toma de decisiones en pacientes asmáticos. Arch Bronconeumol 1999; 35: 435439.

3. Monninkhof EM, van der Valk PD, van der Palen J, van Herwaarden CL, Partidge MR, Walters $\mathrm{EH}$, et al. Self-management education for chronic obstructive pulmonary disease. Cochrane Database Syst Rev 2003; (1): CD002990.

4. Hernandez C, Casas A, Escarrabill J, Alonso J, et al. Home hospitalization of exacerbated copd patients. A randomized controlled trial for clinical efficacy and cost analysis of innovative services for chronically ill patients. Eur Respir J 2003; 21: 58-67.

5. Gallefoss F, Bakke PS. Cost-benefit and cost-effectiveness analysis of selfmanagement in patients with COPD a 1-year follow-up randomized, controlled trial. Respir Med 2002; 96: 424-431.

6. Llor C, Hernandez S. Enfermedad infecciosa en atención primaria: estudio prospectivo efectuado durante todo un año. Enferm Infecc Microbiol Clin 2010; 28(4):222-226.

7. Sobradillo V, Miravitlles M, Gabriel R, Jiménez CA, Villasante C, Masa JF, et al. Geographic variations in prevalence and underdiagnosis of COPD. Results of the IBERPOC multicentric epidemiological study. Chest 2000;118:981-9

8. Miravitlles M, Soriano JB, Munoz L, Garcia Rio F, Sanchez G, Sarmiento M et al.COPD prevalence in Spain in 2007 (EPI-SCAN study results). Eur Resp J 2008; 32(52):308s.

9. Consejería de Salud y Servicios Sanitarios del Principado de Asturias. Guía de Recomendaciones Clínicas. Enfermedad Pulmonar Obstructiva Crónica. Asturias: Dirección General de Organización de las Prestaciones Sanitarias Consejería de Salud y Servicios Sanitarios del Principado de Asturias; 2005.

10. Encuesta nacional de salud. Documento en línea. Con acceso el 4 de diciembre de 2010. Disponible en http://www.ine.es/jaxi/tabla.do

11. Effing T, Monninkhof EM, Van der Valk PDLPM, Van der Palen J, Van Herwaarden CLA, Partidge MR, et al. Educación en autocuidado para pacientes con enfermedad pulmonar obstructiva crónica (Revisión Cochrane traducida). La Biblioteca Cochrane Plus, 2008 Número 2. Oxford: Update Software Ltd. Disponible en: http://www.update-software.com. 
12. Parkes G, Greenhalgh T, Griffin M, Dent R. Effect on smoking quit rate of telling patients their lung age: the Step2quit randomised controlled trial. BMJ 2008; 336:598-600.

13. Herramienta de lectura crítica disponible en: http://www.redcaspe.org/

14. Keranis E, Makris D, Rodopoulou P, Martinou H, Papamakarios G, Daniil Z, et al. Impact of dietary shift to higher-antioxidant foods in COPD: a randomised trial. Eur Respir J. 2010; 36: 774-780.

15. Hirayama F, H Lee A, W Binns CW, Zhao Y, Hiramatsu T, Tanikawa Y, et al. Soy consumption and risk of COPD and respiratory symptons: a case-control study in Japan. Respiratory Research. 2009; 10-56

16. Weekes CE, Emery PW, Elia M. Dietary counseling and food fortification in stable COPD: a randomized trial.Torax.2009; 64: 326-331.

17. Jiang R, Camargo CA, Varraso R, Paik DC, Willett WC, Barr RG. Consumption of cured meats and prospective risk of chronic obstructive pulmonary disease in women. Am J Clin Nutr 2008;87: 1002-1008.

18. Varraso R, Jiang R, Barr RG, Willett WC, Camargo CA. Prospective study of cured meats consumption and risk of chronic obstructive pulmonary disease in men. Am J Epidemiol 2007; 166:1438-1445

19. Varraso R, T Fung T, Barr RG, B Hu F, Willett W, Camargo CA. Prospective study of dietary patterns and chronic obstructive pulmonary disease among US women. Am J Clin Nutr. 2007;86:488-495

20. Varraso R, T Fung T, B Hu F, Willett W, Camargo CA. Prospective study of dietary patterns and chronic obstructive pulmonary disease among US men. Thorax 2007;62:786-791

21. Varraso R, Willett WC, Camargo CA. Prospective Study of Dietary Fiber and Risk of Chronic Obstructive Pulmonary Disease Among US Women and Men. Am J Epidemiol. 2010; 171: 776-784

22. Rosenkranz SK, Townssend DK, Steffens SE, Harms CA. Effects of a high-fat meal on pulmonary function in healthy subjects. Eur J Appl Physiol 2010; 109:499-506

23. Hwa Lee J, Su Sin Y, Young Suh G, Ryu J-S, Ho Shin D, Haeng Koh K et al. Diet and Airway Obstruction: A Cross Sectional Study from the Second Korean National Health and Nutrition Examination Survey. Korean J Intern Med 2010; 25:132-139

24. De Batlle J, Barreiro E, Romieu I, Mendez M, Gómez FP, Balcells E et al. Dietary modulation of oxidative stress in chronic obstructive pulmonary disease patients. Free Radical Research 2010; 44:1296-1303.

25. Carel R, Wan Wetering, Martine Hoogendoorn MSc, Roelinka B, PhD, Gonnie JW et al. Efficacy and Costs of Nutritional Rehabilitation in Muscle-Wasted Patients With Chronic Obstructive Pulmonary Disease in Community-based Setting: A Prespecified Subgroup Analysis of the INTERCOM trial. J Am Med Dir Assoc 2010; 11:179-187.

ISSN 1695-6141

(C) COPYRIGHT Servicio de Publicaciones - Universidad de Murcia 